\title{
Supersymmetric Quantum Mechanics for Bianchi Class A models
}

\author{
J. Socorro* and E. R. Medina ${ }^{\dagger}$ \\ Instituto de Física de la Universidad de Guanajuato, \\ Apartado Postal E-143, C.P. 37150, León, Guanajuato, Mexico.
}

(October 5, 2018)

\begin{abstract}
In this work we present cosmological quantum solutions for all Bianchi Class A cosmological models obtained by means of supersymmetric quantum mechanics. We are able to write one general expression for all bosonic components occuring in the Grassmann expansion of the wave function of the Universe for this class of models. These solutions are obtained by means of a more general ansatz for the so-called master equations.
\end{abstract}

\section{INTRODUCTION}

In recent years, many particular solutions of the Wheeler-DeWitt (WDW) equation in standard quantum cosmology have been found for Bianchi Class A models [1] . In general, the wave functions of these models have the form $\Psi\left(\mathrm{q}^{\mu}\right)=\mathrm{W}\left(\mathrm{q}^{\mu}\right) \mathrm{e}^{ \pm \Phi\left(\mathrm{q}^{\mu}\right)}$, where $\Phi$ is a solution to the corresponding classical Hamilton-Jacobi equation. One can find similar solutions for a WDW equation that has been derived in the bosonic sector of the heterotic string [2]. Some time ago, Graham [3,4] considered the WDW equation in the ADM formulation of the Bianchi type IX model and found the appropriate factorization in terms of the operators which may be considered the square roots of this equation. Graham was the first to provide a master equation for the Bianchi type IX models in Witten's supersymmetric quantum mechanics for the so-called auxiliary function $\mathrm{f}_{ \pm}\left(\mathrm{q}^{\mu}\right)$. However, he studied only the trivial case $\mathrm{f}=$ const. Next, Obregón et al, [5] obtained ans solved the master equation for the Bianchi type II models. For an application of the technique to the 2D Fokker-Planck equation see $\llbracket 7 \rrbracket$.

Our main goal in this work, is to find the general form for all bosonic functions that appear in the Grassmann expansion of the wave function of the Universe, in the approach of Witten's supersymmetric quantum mechanics for all Bianchi Class A cosmological models. In the Grassmannian calculus, one introduces fermionic variable without a direct physical

*E-mail: socorro@ifug2.ugto.mx

†E-mail: emedina@ifug3.ugto.mx 
meaning. Physical quantities of interest, such as $\Psi^{*} \Psi$, require integration over the Grassmannian variables, obtaining in this way information about their influence on the unnormalized probability distributions.

We begin with metrics of the form

$$
\mathrm{ds}^{2}=\left(\mathrm{N}^{2}-\mathrm{N}^{\mathrm{j}} \mathrm{N}_{\mathrm{j}}\right) \mathrm{dt} \mathrm{t}^{2}-2 \mathrm{~N}_{\mathrm{i}} \mathrm{dt} \mathrm{d} \omega^{\mathrm{i}}-\mathrm{e}^{2 \alpha(\mathrm{t})} \mathrm{e}^{2 \beta(\mathrm{t})}{ }_{\mathrm{ij}} \omega^{\mathrm{i}} \omega^{\mathrm{j}}
$$

where $\alpha(\mathrm{t})$ is a scalar and $\beta_{\mathrm{ij}}$ is a $3 \times 3$ matrix, and $\mathrm{N}(\mathrm{t})$ and $\mathrm{N}_{\mathrm{i}}(\mathrm{t})$ are the lapse and shift functions, respectively. The one-forms $\omega^{\mathrm{i}}$ have specific expression for any particular Bianchi Universe [6].

The Arnowitt-Deser-Misner (ADM) action has the form

$$
\mathrm{I}=\int\left(\mathrm{P}_{\mathrm{x}} \mathrm{dx}+\mathrm{P}_{\mathrm{y}} \mathrm{dy}+\mathrm{P}_{\alpha} \mathrm{d} \alpha-\mathrm{N} \mathcal{H}\right) \mathrm{dt}
$$

where

$$
\mathcal{H}=\mathrm{e}^{-3 \alpha}\left(-\mathrm{P}_{\alpha}^{2}+\mathrm{P}_{\mathrm{x}}^{2}+\mathrm{P}_{\mathrm{y}}^{2}+\mathrm{e}^{4 \alpha} \mathrm{V}(\mathrm{x}, \mathrm{y})\right) .
$$

The potential term $\mathrm{e}^{4 \alpha} \mathrm{V}(\mathrm{x}, \mathrm{y})=\mathrm{U}(\alpha, \mathrm{x}, \mathrm{y})$ is specific of the cosmological model under consideration, being related to the function $\Phi\left(q^{\mu}\right)$ as follows [1] $\left(q^{\mu}=(\alpha, x, y)\right)$.

$$
\mathrm{U}\left(\mathrm{q}^{\mu}\right)=\eta^{\mu \nu} \frac{\partial \Phi}{\partial \mathrm{q}^{\mu}} \frac{\partial \Phi}{\partial \mathrm{q}^{\nu}}
$$

This turns into the Einstein-Hamilton-Jacobi equation when we replace the momentum $\mathrm{P}_{\mu} \rightarrow \partial \Phi / \partial \mathrm{q}^{\mu}$ in (3), $\eta^{\mu \nu}=\operatorname{diag}(-1,1,1)$.

In this work we drop the factor $\mathrm{e}^{-3 \alpha}$. This corresponds to fixing the factor ordering before factorizing the WDW equation. The latter is got by replacing $\mathrm{P}_{\mu} \rightarrow-\mathrm{i} \partial_{\mathrm{q}^{\mu}}$ in (3).

The work is organized as follows. In Sec. II we give the factorization of the WDW equation in terms of the supercharges operators and obtain the so-called master equations for an auxiliar function $\mathrm{f}_{ \pm}$in general way for all Bianchi Class A cosmological models. In Sec. III we solve this master equation and present all bosonic components of the wave function for the same models. In Sec. IV, we write a general form for an unnormalized probability density, by imposing some soft of boundary conditions on the bosonic components. Sec. V is devoted to conclusions

\section{FACTORIZATION OF THE WDW EQUATION}

The WDW equation can be obtained as the bosonic sector of a super-Hamiltonian in superspace $\mathrm{H}_{\mathrm{sS}}$ by employing supercharge operators. In the 3D case, the supercharges read

$$
\mathrm{Q}=\psi^{\mu}\left[-\mathrm{P}_{\mu}+\mathrm{i} \frac{\partial \Phi}{\partial \mathrm{q}^{\mu}}\right],
$$

and

$$
\overline{\mathrm{Q}}=\bar{\psi}^{\nu}\left[-\mathrm{P}_{\nu}-\mathrm{i} \frac{\partial \Phi}{\partial \mathrm{q}^{\nu}}\right],
$$


with the following algebra for the variables $\psi^{\mu}$ and $\bar{\psi}^{\nu}$,

$$
\left\{\psi^{\mu}, \bar{\psi}^{\nu}\right\}=\eta^{\mu \nu}, \quad\left\{\psi^{\mu}, \psi^{\nu}\right\}=0, \quad\left\{\bar{\psi}^{\mu}, \bar{\psi}^{\nu}\right\}=0 .
$$

Using the representations $\psi^{\mu}=\eta^{\mu \nu} \partial / \partial \theta^{\nu}$ and $\bar{\psi}^{\nu}=\theta^{\nu}$, one finds the superspace Hamiltonian in the form

$$
\mathrm{H}_{\mathrm{ss}}=\frac{1}{2}\{\mathrm{Q}, \overline{\mathrm{Q}}\}=\mathcal{H}_{0}+\frac{\hbar}{2} \frac{\partial^{2} \Phi}{\partial \mathrm{q}^{\mu} \partial \mathrm{q}^{\nu}}\left[\bar{\psi}^{\mu}, \psi^{\nu}\right]
$$

where $\mathcal{H}_{0}=\square+U\left(q^{\mu}\right)$ is the standard WDW equation, $\square$ is the 3D d'Alambertian in the $\mathrm{q}^{\mu}$ coordinates with signature $(-++),\{$,$\} means the anti-commutator, and [, ], the$ commutator. Notice that the quantized super-Hamiltonian differs from the classical one $\mathcal{H}_{0}$ by a spin term, which vanishes in the classical limit.

The supercharges $\mathrm{Q}, \overline{\mathrm{Q}}$ and the super-Hmiltonian satisfy the following algebra

$$
\frac{1}{2}\{Q, \bar{Q}\}=H_{s s}, \quad\left\{H_{s s}, Q\right\}=0, \quad\left\{H_{s s}, \bar{Q}\right\}=0 .
$$

Physical states are selected by the constraints

$$
Q \Psi=0, \quad \bar{Q} \Psi=0,
$$

where the following 3D Grassmann representation of the $\Psi$ is used

$$
\Psi=\mathcal{A}_{+}+\mathcal{B}_{\nu} \theta^{\nu}+\frac{1}{2} \epsilon_{\mu \nu \lambda} \mathcal{C}^{\lambda} \theta^{\mu} \theta^{\nu}+\mathcal{A}_{-} \theta^{0} \theta^{1} \theta^{2}
$$

$\mu, \nu, \lambda$ running over $0,1,2$.

The following ansatz

$$
\mathcal{B}_{\mu}=\frac{\partial \mathrm{f}_{+}}{\partial \mathrm{q}^{\mu}} \mathrm{e}^{-\Phi}
$$

in (10) and (11), leads to the master equation for the auxiliary function $\mathrm{f}_{+}$

$$
\mathrm{uf}_{+}-2 \eta^{\mu \nu} \frac{\partial \Phi}{\partial \mathrm{q}^{\mu}} \frac{\partial \mathrm{f}_{+}}{\partial \mathrm{q}^{\nu}}=0
$$

In addition, it is possible to show that $\frac{1}{2} \epsilon_{\mu \nu \lambda} \mathcal{C}^{\lambda} \theta^{\alpha} \theta^{\mu} \theta^{\nu}=\mathcal{C}^{\alpha} \theta^{0} \theta^{1} \theta^{2}$ and employing the ansatz

$$
\mathcal{C}^{\alpha}=\eta^{\alpha \mu} \frac{\partial \mathrm{f}_{-}}{\partial \mathrm{q}^{\mu}} \mathrm{e}^{\Phi}
$$

we obtain the second master equation of the form

$$
\mathrm{ff}_{-}+2 \eta^{\mu \nu} \frac{\partial \Phi}{\partial \mathrm{q}^{\mu}} \frac{\partial \mathrm{f}_{-}}{\partial \mathrm{q}^{\nu}}=0 .
$$

Thus, (13) and (15) can be rewritten as

$$
\square \mathrm{f}_{ \pm} \mp 2 \eta^{\mu \nu} \frac{\partial \Phi}{\partial \mathrm{q}^{\mu}} \frac{\partial \mathrm{f}_{ \pm}}{\partial \mathrm{q}^{\nu}}=0 .
$$

The solutions for the other functions are

$$
\mathcal{A}_{ \pm}=q_{ \pm} e^{\mp \Phi}
$$

where $\mathrm{q}_{ \pm}$are integration constants.

In the following, we give the solution of (16), using as toy models the Bianchi Class A cosmological models [1] 


\section{QUANTUM SOLUTIONS FOR BIANCHI CLASS A MODELS}

To solve (16) it is necessary to know the potentials $\mathrm{U}\left(\mathrm{q}^{\mu}\right)$ (or $\Phi\left(q^{\mu}\right)$ ) for the Bianchi Class A cosmological models. These are given in table I [1]

\begin{tabular}{|c|c|c|}
\hline Bianchi type & Potential U & $\Phi$ \\
\hline $\mathrm{I}$ & 0 & 0 \\
\hline II & $\frac{1}{3} e^{4 \beta_{1}}$ & $\pm \frac{1}{6} \mathrm{e}^{2 \beta_{1}}$ \\
\hline $\mathrm{VI}_{\mathrm{h}=-1}$ & $\frac{4}{3} e^{2\left(\beta_{1}+\beta_{2}\right)}$ & $\pm \frac{1}{6}\left[2\left(\beta_{1}-\beta_{2}\right)\right] \mathrm{e}^{\beta_{1}+\beta_{2}}$ \\
\hline $\mathrm{VII}_{\mathrm{h}=0}$ & \begin{tabular}{|l|l|}
$\frac{1}{3}$ & $e^{4 \beta_{1}}+e^{4 \beta_{2}}-2 e^{2\left(\beta_{1}+\beta_{2}\right)}$ \\
\end{tabular} & \begin{tabular}{|l|l|}
$\pm \frac{1}{6}$ & $\mathrm{e}^{2 \beta_{1}}+\mathrm{e}^{2 \beta_{2}}$ \\
\end{tabular} \\
\hline VIII & $\frac{1}{3} e^{4 \beta_{1}}+e^{4 \beta_{2}}+e^{4 \beta_{3}}-2 e^{2\left(\beta_{1}+\beta_{2}\right)}+2 e^{2\left(\beta_{1}+\beta_{3}\right)}+2 e^{2\left(\beta_{2}+\beta_{3}\right)}$ & $\pm \frac{1}{6} \mathrm{e}^{2 \beta_{1}}+\mathrm{e}^{2 \beta_{2}}-\mathrm{e}^{2 \beta_{3}}$ \\
\hline IX & $\frac{1}{3} \mid e^{4 \beta_{1}}+e^{4 \beta_{2}}+e^{4 \beta_{3}}+2 e^{2\left(\beta_{1}+\beta_{2}\right)}+2 e^{2\left(\beta_{1}+\beta_{3}\right)}+2 e^{2\left(\beta_{2}+\beta_{3}\right)}$ & $\pm \frac{1}{6} \quad \mathrm{e}^{2 \beta_{1}}+\mathrm{e}^{2 \beta_{2}}+\mathrm{e}^{2 \beta_{3}}$ \\
\hline
\end{tabular}

Potential $\mathrm{U}$ and superpotential $\Phi$ for all Bianchi Class A models, where

$$
\beta_{1}=\alpha+\mathrm{x}+\sqrt{3} \mathrm{y}, \beta_{2}=\alpha+\mathrm{x}-\sqrt{3} \mathrm{y} \text { and } \beta_{3}=\alpha-2 \mathrm{x} .
$$

Once $\mathrm{f}_{ \pm}$are obtained, all the bosonic components that appear in the Grassmann expansion of the wave function (11) can be determined as follows. The ansatz

$$
\mathrm{f}_{ \pm}\left(\mathrm{q}^{\mu}\right)=\mathrm{W}_{ \pm}\left(\mathrm{q}^{\mu}\right) \mathrm{e}^{ \pm \Phi\left(\mathrm{q}^{\mu}\right)} .
$$

turns (16) into

$$
\square \mathrm{W}_{ \pm}=\left[(\nabla \Phi)^{2} \mp \square \Phi\right] \mathrm{W}_{ \pm}
$$

whose solutions are

$$
\mathrm{W}_{ \pm}\left(\mathrm{q}^{\mu}\right)=\beta_{ \pm} \mathrm{e}^{\mathrm{m}_{\mu} \mathrm{t}^{\mu}} \mathrm{e}^{\mp \Phi}
$$

where $\beta_{ \pm}$are integration constants, $\mathrm{m}_{\mu}=\left(\mathrm{m}_{1}, \mathrm{~m}_{2}, \mathrm{~m}_{3}\right)$ is a vector of null measure (i.e. $\left.-\mathrm{m}_{1}^{2}+\mathrm{m}_{2}^{2}+\mathrm{m}_{3}^{2}=0\right)$ and $\mathrm{t}^{\mu}=(-\alpha, \mathrm{x}, \mathrm{y})$.

Thus, the solutions for $f_{ \pm}$are given by

$$
\mathrm{f}_{ \pm}=\beta_{ \pm} \mathrm{e}^{\mathrm{m}_{\mu} \mathrm{t}^{\mu}}
$$

In the Table II, we write the corresponding $\mathrm{m}_{\mu} \mathrm{t}^{\mu}$ terms for all Bianchi Class A models.

\begin{tabular}{|l|c|c|}
\hline Bianchi type & $\mathrm{m}_{\mu} \mathrm{t}^{\mu}=-\mathrm{m}_{1_{ \pm}} \alpha+\mathrm{m}_{2_{ \pm}} \mathrm{x}+\mathrm{m}_{3_{ \pm}} \mathrm{y}$ & $\mathrm{m}_{\mu}$ \\
\hline $\mathrm{I}$ & $-\mathrm{m}_{1_{ \pm}} \alpha+\mathrm{m}_{2_{ \pm}} \mathrm{x}+\mathrm{m}_{3_{ \pm}} \mathrm{y}$ & $\left(m_{1_{ \pm}}, m_{2_{ \pm}}, m_{3_{ \pm}}\right)$ \\
\hline $\mathrm{II}$ & $\mathrm{b}_{ \pm}(2 \alpha-\mathrm{x}+\sqrt{3} \mathrm{y})$ & $\mathrm{b}_{ \pm}(-2,-1, \sqrt{3})$ \\
\hline $\mathrm{VI}_{\mathrm{h}=-1}$ & $\mathrm{~b}_{ \pm}(\alpha+\mathrm{x})$ & $\mathrm{b}_{ \pm}(-1,1,0)$ \\
\hline $\mathrm{VII}$ & $\mathrm{b}_{ \pm}(\alpha+\mathrm{x})$ & $\mathrm{b}_{ \pm}(-1,1,0)$ \\
\hline $\mathrm{VIII}$ & 0 & $(0,0,0)$ \\
\hline $\mathrm{IX}$ & 0 & $(0,0,0)$ \\
\hline
\end{tabular}

Table II

$\mathrm{m}_{\mu} \mathrm{t}^{\mu}$ and $\mathrm{m}_{\mu}$ terms for all Bianchi Class A models. 
Moreover, the solutions for all bosonic components of the wave function are

$$
\begin{array}{rlrl}
\mathcal{A}_{ \pm} & =\mathrm{q}_{ \pm} \mathrm{e}^{\mp \Phi} & \\
\mathcal{B}_{0}=-\mathrm{m}_{1_{+}} \mathrm{f}_{+} \mathrm{e}^{-\Phi}, & \mathcal{C}^{0}=\mathrm{m}_{1_{-}} \mathrm{f}_{-} \mathrm{e}^{\Phi} \\
\mathcal{B}_{1}=\mathrm{m}_{2_{+}} \mathrm{f}_{+} \mathrm{e}^{-\Phi}, & \mathcal{C}^{1}=\mathrm{m}_{2_{-}} \mathrm{f}_{-} \mathrm{e}^{\Phi} \\
\mathcal{B}_{2}=\mathrm{m}_{3_{+}} \mathrm{f}_{+} \mathrm{e}^{-\Phi}, & \mathcal{C}^{2}=\mathrm{m}_{3_{-}} \mathrm{f}_{-} \mathrm{e}^{\Phi}
\end{array}
$$

where $\mathrm{q}_{ \pm} a n d b_{ \pm}$are arbitrary constants and $\mathrm{m}_{\mathrm{i}_{ \pm}}$are constants given in table II.

\section{THE UNNORMALIZED PROBABILITY DENSITY $|\Psi|^{2}$}

In this section we follow ref. [5] to get $|\Psi|^{2}$ for the wave function given by (11). Integrating over the Grassmann variables $\theta^{\mu}[\mathbb{B}]$, we are led to the following result

$$
|\Psi|^{2}=\mathcal{A}_{+}^{*} \mathcal{A}_{+}+\mathcal{B}_{0}^{*} \mathcal{B}_{0}+\mathcal{B}_{1}^{*} \mathcal{B}_{1}+\mathcal{B}_{2}^{*} \mathcal{B}_{2}+\mathcal{C}^{0 *} \mathcal{C}^{0}+\mathcal{C}^{1 *} \mathcal{C}^{1}+\mathcal{C}^{2 *} \mathcal{C}^{2}+\mathcal{A}_{-}^{*} \mathcal{A}_{-} .
$$

Assuming that the constants appearing in (23) are real, we obtain

$$
|\Psi|^{2}=\left[\mathrm{q}_{+}^{2}+\left(\mathrm{m}_{1_{+}}^{2}+\mathrm{m}_{2_{+}}^{2}+\mathrm{m}_{3_{+}}^{2}\right) \mathrm{f}_{+}^{2}\right] \mathrm{e}^{-2 \Phi}+\left[\mathrm{q}_{-}^{2}+\left(\mathrm{m}_{1_{-}}^{2}+\mathrm{m}_{2_{-}}^{2}+\mathrm{m}_{3_{-}}^{2}\right) \mathrm{f}_{-}^{2}\right] \mathrm{e}^{2 \Phi} .
$$

For example, for the Bianchi type II models, we obtain from (24)

$$
\left|\Psi_{\mathrm{II}}\right|^{2}=\left(\mathrm{q}_{+}^{2}+8 \mathrm{~b}_{+}^{2} \mathrm{f}_{+}^{2}\right) \mathrm{e}^{-2 \Phi}+\left(\mathrm{q}_{-}^{2}+8 \mathrm{~b}_{-}^{2} \mathrm{f}_{-}^{2}\right) \mathrm{e}^{2 \Phi},
$$

which is Eq. (4.2) in [5].

Now, by imposing the some sort of boundary conditions, only one kind of terms will remain in the wave function [9]. There are many possibilities, one is to demand that $\Psi$ does not diverge for $|\mathrm{x}|,|\mathrm{y}| \rightarrow \infty$ at fixed $\alpha$. In such a case only the terms $\mathrm{e}^{-\Phi}$ will remain in (24).

Figures 1 and 2, display the behavior of the probabilitity density $|\Psi|^{2}$ for the Bianchi type $\mathrm{VI}_{h=-1}$ and $\mathrm{VII}_{h=0}$ when the parameter $\mathrm{b}_{+}=-1$, respectively. The $|\Psi|^{2}$ for the same models when $b_{+}=+1$ are plotted in Figures 3 and 4 . The $|\Psi|^{2}$ behavior for the Bianchi type II and IX, has been given in [5] and [3], respectively.

\section{CONCLUSION}

Our main goal in this work, was to obtain a general expression for all bosonic functions that appear in the expansion of the wave function of the Universe using the approach of Witten's supersymmetric quantum mechanics. This has been done for all Bianchi Class A cosmological models. In addition, we found the general form for the unnormalized probabilitity density, (24), for any Bianchi Class A cosmological model. When we apply boundary conditions on the wave function, the unnormalized probability density is reduced to

$$
|\Psi|^{2}=\left[\mathrm{q}_{+}^{2}+\left(\mathrm{m}_{1_{+}}^{2}+\mathrm{m}_{2_{+}}^{2}+\mathrm{m}_{3_{+}}^{2}\right) \mathrm{f}_{+}^{2}\right] \mathrm{e}^{-2 \Phi} .
$$

At this step, the main contribution to $|\Psi|^{2}$ will depend on the values of the parameter $\mathrm{b}_{+}$that occurs in the function $\mathrm{f}_{+}$and on the range used for the coordinates $\alpha$ and $(\mathrm{x}, \mathrm{y})$. 


\section{ACKNOWLEDGMENTS}

We would like to thank H. Rosu for valuable hints. This research was supported by CONACyT. ERM was supported by a CONACYT student fellowship. 


\section{REFERENCES}

[1] O. Obregón and J. Socorro, Int. J. Theor. Phys. 35, 1381 (1996). For review, see A. Macías, Gen. Rel. and Grav. 31, 653 (1999).

[2] J.E. Lidsey, Phys. Rev. D 49 R599 (1994).

[3] R. Graham, Phys. Rev. Lett. 67, 1381 (1991).

[4] R. Graham, Phys. Rev. D 48, 1602 (1993).

[5] O. Obregón, J. Socorro and J. Benítez, Phys. Rev. D 47, 4471 (1993).

[6] M.P. Ryan and L.C. Shepley, Homogeneous Relativistic Cosmologies, (Princeton University Press, Princeton, NJ 1975).

[7] H.C. Rosu, J. Socorro and O. Obregón, J. Phys. A: Math. Gen. 29, 1825 (1996).

[8] L.D. Faddeev and A.A. Slavnov, Gauge Fields: An Introduction to Quantum Theory (Addison-Wesley, Reading, MA. 1991), sec. 2.5.

[9] S.W. Hawking and D.N. Page, Phys. Rev. D 42, 2655 (1990). 

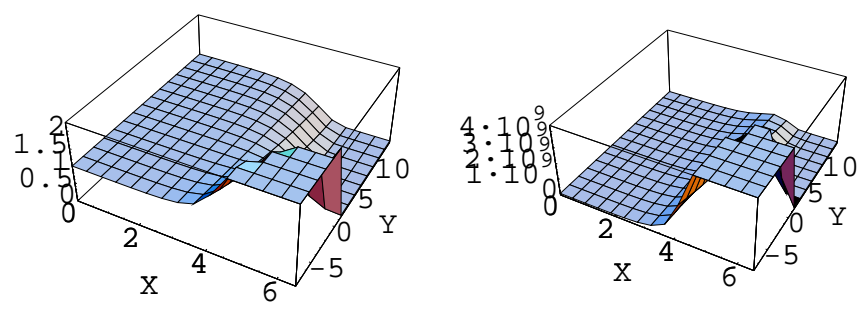

Fig. 1

At the left we show $\left|\Psi_{\mathrm{VI}}\right|^{2}=\mathrm{q}_{+}^{2} \mathrm{e}^{-2 \Phi}$, for the first pure bosonic term, when $\mathrm{q}_{+}=1, \alpha=-6$. At the right, the main contribution to the unnormalized probability density due to the $\mathcal{B}_{\mu}$ terms, when $\beta_{+}=1, b_{+}=-1, \alpha=-6$.
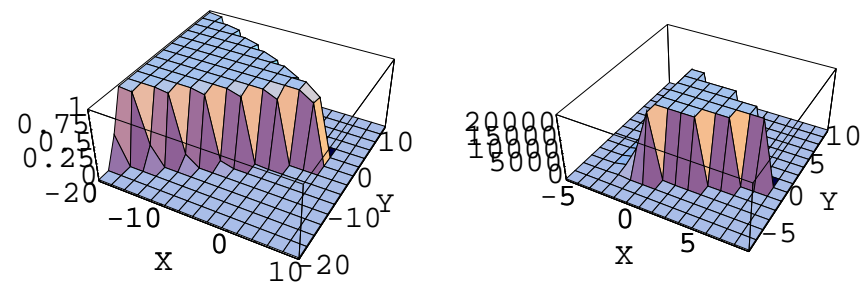

Fig. 2

At the left we show $\left|\Psi_{\mathrm{VII}}\right|^{2}=\mathrm{q}_{+}^{2} \mathrm{e}^{-2 \Phi}$, for the first pure bosonic term when $\mathrm{q}_{+}=1, \alpha=-6$. At the right, the main contribution to the unnormalized probability density due to the $\mathcal{B}_{\mu}$ terms, when $\beta_{+}=1, b_{+}=-1, \alpha=-6$. 

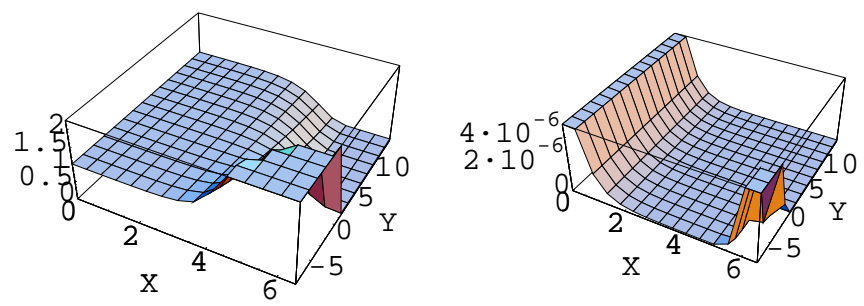

Fig. 3

At the left we show $\left|\Psi_{\mathrm{VI}}\right|^{2}=\mathrm{q}_{+}^{2} \mathrm{e}^{-2 \Phi}$, the main contribution to the unnormalized probability density due to the first pure bosonic term, when $\mathrm{q}_{+}=1, \alpha=-6$. At the right, the corresponding contribution of the $\mathcal{B}_{\mu}$ terms, for $\beta_{+}=1, b_{+}=1, \alpha=-6$.
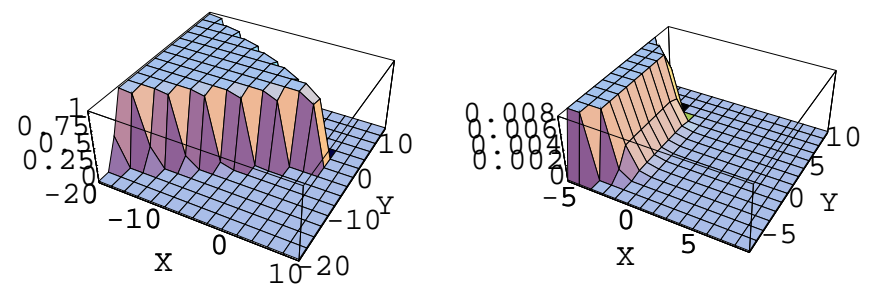

Fig. 4

At the left we show $\left|\Psi_{\mathrm{VII}}\right|^{2}=\mathrm{q}_{+}^{2} \mathrm{e}^{-2 \Phi}$, the main contribution to the unnormalized probability density due to the first pure bosonic term, when $\mathrm{q}_{+}=1, \alpha=-6$. At the right, the corresponding contribution of the $\mathcal{B}_{\mu}$ terms, for $\beta_{+}=1, b_{+}=1, \alpha=-6$. 